\title{
INFLUENCE OF PRE-TENSION ON TORSION OF MICROSCALE CU WIRES: A STUDY VIA STRAIN GRADIENT THEORY
}

\author{
Xinbing MA, JinXing LiU \\ Jiangsu University, Faculty of Civil Engineering and Mechanics, Zhenjiang, Jiangsu Province, People's Republic of China \\ e-mail: jxliu@mails.ucas.ac.cn
}

Nonproportional plastic deformations on the microscale are an emerging topic. A simplified theory of strain gradient elasto-plasticity is developed to study the evolution of yield strength in a copper wire sequentially experiencing tension and torsion. The pre-tension deformation and stress are inherited to the upcoming torsion process, resulting in a nonproportional loading condition. With consideration of the extra hardening effect due to strain gradient, pre-tension weakens the extra hardening effect of the strain gradient and the dependence on the wire radius. Cyclic torsion behavior is also investigated. Anomalous Bauschinger effect and plastic softening are found.

Keywords: nonproportional loading, strain gradient plasticity, size effect, cyclic response

\section{Introduction}

Plastic deformations on the micro scale have some featuers which are distinctively different from those on the macroscale. For example, in tests of wire torsion (e.g. Fleck et al., 1994; Liu et al., 2013a,b), microbend (e.g. Stölken and Evans, 1998) and indentation (e.g. Nix and Gao, 1998), the yield strength shows a considerable dependence on the specimen size while material is the same and grain sizes are similar. This size effect is often regarded contributed by geometrically necessary dislocations (GND) and impossible to be modeled based on the conventional plasticity (e.g. Fleck et al., 1994; Gao and Huang, 2003). Moreover, there are several other characteristics related to the above size effect, such as the size effect around the elastic limit (e.g. Chakravarthy and Curtin, 2011; Dunstan et al., 2009; Liu et al., 2013a,b), anomalous Bauschinger effect (e.g. Bardella and Panteghini, 2015; Fertig and Baker, 2009; Idiart and Fleck, 2010; Kiener et al., 2010; Liu et al., 2013a,b, 2015; Nicola et al., 2006; Xiang and Vlassak, 2006), plastic softening and unconventional load-unload hysteresis loops (e.g. Kiener et al., 2010).

SGP theories are among the efforts to model the above unconventional effects. Intrinsic scale parameters are included in the constitutive relation in order to consider the extra hardening due to GND (e.g. Fleck and Hutchinson, 1997, 2001; Fleck et al., 1994; Fleck and Willis, 2009; Forest and Sievert, 2003; Gao and Huang, 2001; Gudmundson, 2004). Strain gradient elasto-plasticity (SGEP) (Liu and Soh, 2016), different from many existing SGPs in which elastic deformation is completely ignored or rarely considered, can catch all the above non-classical characteristics. Such a SGEP satisfies thermodynamical conditions. Furthermore, the elastic strain gradient does not change the linear-elastic stiffness during the elastic stage, and plays its role in altering the yield strength only after the plastic deformation has begun. SGEP by Liu and Soh (2016) is free of the issue in the SGP theory by Fleck et al. (1994) and Fleck and Hutchinson (1997), where the unphysical dependence of the initial slope of the torque-twist relation on the plastic length exists. We believe that this issue is mainly due to their assumption that the deformation is entirely plastic from the very beginning, i.e. the rigid-plastic assumption. Liu and Soh (2016) 
took the elastic strain and strain gradient as parts of recoverable deformations and accounted for their contributions to hardening.

One remaining challenge in this field is the modeling of microscale plastic deformations under nonproportional loading conditions (e.g. Fleck et al., 2014; Janssens, 2018; Ozhoga-Maslovskaja et al., 2015; Pejkowski, 2017; Yang and Vormwald, 2017). Thus, we make an attempt to meet this challenge based on a simplified version of the SGEP (Liu and Soh, 2016), where one single intrinsic scale parameter appears.

The paper is organized as follows. In Section 2, the theoretical formulation of the simplified SGEP is constructed, with only one plastic length and without higher order stresses. In Section 3, the analytical solution is provided for torsion of a pre-tension wire. In Section 4, numerical results show the effect of pre-tension on torsion of microscale $\mathrm{Cu}$ wires. The paper ends with conclusions in Section 5 .

\section{Theoretical formulation}

Strain gradient elasto-plasticity (SGEP) adopted here was initially built aiming to predict unconventional features like size effects and anomalous Bauschinger effect on the microscale (Liu and Soh, 2016). Its incremental form is summarized as follows.

The strain rate and the rate of the second-order gradient of the displacement $\dot{u}_{i}$ are respectively,

$$
\dot{\varepsilon}_{i j}=\frac{1}{2}\left(\partial_{i} \dot{u}_{j}+\partial_{j} \dot{u}_{i}\right) \quad \dot{\eta}_{i j k}=\partial_{i j} \dot{u}_{k}
$$

Based on the fact that the elastic lengths are generally far smaller than the plastic ones, one conclusion is that the strain gradient is dominantly elastic out of the elasto-plastic decomposition according to the associate rule. The other is that omitting the elastic length scales and, therefore, higher-order stresses is reasonable. This simplification leads to substantial reduction in the complexity of formulations and calculations, nevertheless meanwhile good agreements with experimental data can still be achieved.

$\dot{\varepsilon}_{i j}$ is divided into a deviatoric part $\dot{\varepsilon}_{i j}^{\prime}$ and a volumetric part $\dot{\varepsilon}_{k k} . \dot{\eta}_{i j k}$ is purely elastic. Then, the deviatoric part $\dot{\varepsilon}_{i j}^{\prime}$ is decomposed into an elastic and plastic part

$$
\dot{\varepsilon}_{i j}^{\prime}=\dot{\varepsilon}_{i j}^{\prime}+\dot{\varepsilon}_{i j}^{p}
$$

Hooke's law serves as the elastic constitutive law, and relates stress to elastic deformation, i.e.

$$
\sigma_{i j}=\lambda \delta_{i j} \varepsilon_{m m}^{e}+2 \mu \varepsilon_{i j}^{e}
$$

And the total effective stress is simply contributed to the Cauchy stress $\sigma_{i j}$ due to negligence of higher order stresses, i.e.

$$
\sigma_{e}^{t}=\sigma_{e}=\sqrt{\frac{3}{2} \sigma_{i j}^{\prime} \sigma_{i j}^{\prime}}
$$

The total effective elasto-plastic strain $\varepsilon_{e}^{t, e p}$ is defined as by Liu and Soh (2016)

$$
\varepsilon_{e}^{t, e p}=\left[\left(\varepsilon_{e}^{e}+\varepsilon_{e}\right)^{2 \beta}+l\left(\eta_{e}^{e}+\eta_{e}\right)^{2 \beta}\right]^{\frac{1}{2 \beta}}
$$

where

$$
\begin{array}{rlrl}
\varepsilon_{e}^{e} & =\int \sqrt{\frac{2}{3} \dot{\varepsilon}_{i j}^{\prime} \dot{\varepsilon}_{i j}^{\prime}} d t & \varepsilon_{e} & =\int \sqrt{\frac{2}{3} \dot{\varepsilon}_{i j}^{p} \dot{\varepsilon}_{i j}^{p}} d t \\
\eta_{e}^{e} & =\int \sqrt{\frac{2}{3} \dot{\eta}_{i j k}^{e} \dot{\eta}_{i j k}^{e}} d t & \eta_{e}=\int \sqrt{\frac{2}{3} \dot{\eta}_{i j k}^{p} \dot{\eta}_{i j k}^{p}} d t
\end{array}
$$


and $\beta$ is a constitutive parameter to determine how the effective strain and strain gradient are combined to form $\varepsilon_{e}^{t, e p} ; l$ is the plastic characteristic length. In this study, we assume that $l$ works for both recoverable GNDs (energetic) and unrecoverable GNDs (dissipative). From Eq. (2.5), strain gradient has an extra hardening effect, lifting the yield strength.

Notably, in Eq. (2.5) we have not decomposed the strain gradient. This is different from the counterpart in Liu and Soh (2016).

Besides, the hardening law can be expressed as

$$
\sigma^{t}= \begin{cases}\sigma_{Y}+\left(\sigma_{0}^{t}-\sigma_{Y}\right) \frac{\varepsilon_{e}^{t, e p}}{\varepsilon_{0}^{t}} & \text { if } \varepsilon_{e}^{t, e p} \leqslant \varepsilon_{0}^{t} \\ \sigma_{0}^{t}\left(\frac{\varepsilon_{e}^{t, e p}}{\varepsilon_{0}^{t}}\right)^{N} & \text { if } \varepsilon_{e}^{t, e p}>\varepsilon_{0}^{t}\end{cases}
$$

with

$$
\varepsilon_{0}^{t}=\left(\frac{\varepsilon_{e}^{t, e p}}{\varepsilon_{e}^{e}+\varepsilon_{e}}\right)^{\gamma_{\varepsilon}} \varepsilon_{Y} \quad \sigma_{0}^{t}=\left(\frac{\varepsilon_{e}^{t, e p}}{\varepsilon_{e}^{e}+\varepsilon_{e}}\right)^{\gamma_{\sigma}} \sigma_{Y}
$$

where the readers are referred to Liu and Soh (2016) for details.

\section{Circular wire in torsion and tension}

Suppose that the wire of radius $a$, which is lying along the $x_{3}$ axis of a Cartesian co-ordinate system $\left(x_{1}, x_{2}, x_{3}\right)$, is stretched until it reaches deformation of a certain degree $\bar{\varepsilon}$; thereafter, it is twisted, with further axial deformation prevented. Eleftheriadis et al. (2012) conducted a similar investigation. In the following, we solve the problem by dividing it into the tension and torsion stages, respectively.

\subsection{Tension stage}

During the whole tension process, the strain gradient $\eta$ remains zero.

The nonzero strain increments are

$$
\dot{\varepsilon}_{33}=\dot{\bar{\varepsilon}} \quad \dot{\varepsilon}_{11}=\dot{\varepsilon}_{22}=-\frac{1}{2} \dot{\varepsilon}_{33}
$$

where the deformation is assumed to be incompressible.

The strain increments are decomposed into elastic and plastic parts as follows

$$
\dot{\bar{\varepsilon}}=\dot{\bar{\varepsilon}}^{e}+\dot{\bar{\varepsilon}}^{p} \quad \dot{\varepsilon}_{33}=\dot{\varepsilon}_{33}^{e}+\dot{\varepsilon}_{33}^{p} \quad \dot{\varepsilon}_{11}=\dot{\varepsilon}_{11}^{e}+\dot{\varepsilon}_{11}^{p} \quad \dot{\varepsilon}_{22}=\dot{\varepsilon}_{22}^{e}+\dot{\varepsilon}_{22}^{p}
$$

where the relations among above components can be written as

$$
\begin{array}{lll}
\dot{\varepsilon}_{33}^{e}=\dot{\bar{\varepsilon}}^{e} & \dot{\varepsilon}_{33}^{p}=\dot{\bar{\varepsilon}}^{p} & \dot{\varepsilon}_{11}^{e}=\dot{\varepsilon}_{22}^{e}=-\frac{1}{2} \dot{\varepsilon}_{33}^{e} \\
\dot{\varepsilon}_{e}^{e}=\dot{\varepsilon}_{33}^{e} & \dot{\varepsilon}_{e}=\dot{\varepsilon}_{33}^{p} &
\end{array}
$$

The non-vanishing stress components and the effective stress are respectively

$$
\sigma_{33}=\sigma_{e}=E \bar{\varepsilon}^{e}
$$

where $\bar{\varepsilon}^{e}=\int_{0}^{t} \dot{\bar{\varepsilon}}^{e} d t$.

In Liu and Soh (2016), the total effective stress was defined as a combination of the Cauchy stress and the higher order stress. Here, due to neglecting the higher order stress, there is

$$
\sigma_{e}^{t}=\sigma_{e}=E \bar{\varepsilon}^{e}
$$


By keeping in mind that no strain gradient appears, the total effective strain (Liu and Soh, 2016) under monotonic tension is

$$
\varepsilon_{e}^{t, e p}=\varepsilon_{e}^{e}+\varepsilon_{e}=\varepsilon_{33}
$$

Based on Eq. (3.3) and (3.6), the reference strain and stress are, respectively, expressed as

$$
\varepsilon_{0}^{t}=\left(\frac{\varepsilon_{e}^{t, e p}}{\varepsilon_{e}^{e}+\varepsilon_{e}}\right)^{\gamma_{\varepsilon}} \varepsilon_{Y}=\varepsilon_{Y} \quad \sigma_{0}^{t}=\left(\frac{\varepsilon_{e}^{t, e p}}{\varepsilon_{e}^{e}+\varepsilon_{e}}\right)^{\gamma_{\sigma}} \sigma_{Y}=\sigma_{Y}
$$

The yield initiation occurs when

$$
\varepsilon_{e}^{t, e p}=\varepsilon_{Y}
$$

\subsection{Torsion stage}

After the total tensile strain reaches a certain value $\bar{\varepsilon}$, the wire elongation is stopped from further increasing, and the wire is twisted around the $x_{3}$ axis. The twist per unit length is $\kappa$. During the present stage, the strain gradient $\eta$ is non-zero.

Considering the loading and unloading cycles, we continue to formulate the torsion process in an incremental form. The displacement field is

$$
\dot{u}_{1}=-\dot{\kappa} x_{2} x_{3} \quad \dot{u}_{2}=-\dot{\kappa} x_{1} x_{3} \quad \dot{u}_{3}=0
$$

The associated non-vanishing components of the strain increment $\dot{\varepsilon}_{i j}$ and strain gradient increment $\dot{\eta}_{i j k}$ are respectively

$$
\begin{array}{ll}
\dot{\varepsilon}_{13}=\dot{\varepsilon}_{31}=-\frac{\dot{\kappa}}{2} x_{2} & \dot{\varepsilon}_{23}=\dot{\varepsilon}_{32}=-\frac{\dot{\kappa}}{2} x_{1} \\
\dot{\eta}_{231}=\dot{\eta}_{321}=-\dot{\kappa} & \dot{\eta}_{132}=\dot{\eta}_{312}=\dot{\kappa}
\end{array}
$$

At each radial position, $\kappa$ is decomposed into the elastic and plastic part

$$
\dot{\kappa}=\left|\dot{\kappa}_{\varepsilon}^{e}\right|+\dot{\kappa}_{\varepsilon}^{p} \operatorname{sgn}(\dot{\kappa})=\left|\dot{\kappa}_{\eta}^{e}\right|+\dot{\kappa}_{\eta}^{p} \operatorname{sgn}(\dot{\kappa})
$$

Correspondingly, the strain increment $\dot{\varepsilon}_{i j}$ and strain gradient increment $\dot{\eta}_{i j k}$ are both decomposed into the elastic and plastic part

$$
\begin{array}{llr}
\dot{\varepsilon}_{13}^{e}=\dot{\varepsilon}_{31}^{e}=-\frac{\dot{\kappa}_{\varepsilon}^{e}}{2} x_{2} & \dot{\varepsilon}_{23}^{e}=\dot{\varepsilon}_{32}^{e}=-\frac{\dot{\kappa}_{\varepsilon}^{e}}{2} x_{1} & \dot{\varepsilon}_{e}^{e}=\frac{\dot{\kappa}_{\varepsilon}^{e} r}{\sqrt{3}} \\
\dot{\varepsilon}_{13}^{p}=\dot{\varepsilon}_{31}^{p}=-\frac{\dot{\kappa}_{\varepsilon}^{p}}{2} x_{2} & \dot{\varepsilon}_{23}^{p}=\dot{\varepsilon}_{32}^{p}=-\frac{\dot{\kappa}_{\varepsilon}^{p}}{2} x_{1} & \dot{\varepsilon}_{e}=\frac{\dot{\kappa}_{\varepsilon}^{p} r}{\sqrt{3}} \\
\dot{\eta}_{231}^{e}=\dot{\eta}_{321}^{e}=-\dot{\kappa}_{\eta}^{e} & \dot{\eta}_{132}^{e}=\dot{\eta}_{312}^{e}=\dot{\kappa}_{\eta}^{e} & \dot{\eta}_{e}^{e}=\dot{\kappa}_{\eta}^{e} \\
\dot{\eta}_{231}^{p}=\dot{\eta}_{321}^{p}=-\dot{\kappa}_{\eta}^{p} & \dot{\eta}_{132}^{p}=\dot{\eta}_{312}^{p}=\dot{\kappa}_{\eta}^{p} & \dot{\eta}_{e}=\dot{\kappa}_{\eta}^{p}
\end{array}
$$

The non-vanishing stress components and the effective stress are respectively

$$
\begin{aligned}
& \sigma_{13}=\sigma_{31}=-\mu \kappa_{\varepsilon}^{e} x_{2} \quad \sigma_{23}=\sigma_{32}=\mu \kappa_{\varepsilon}^{e} x_{1} \quad \sigma_{33}=E \bar{\varepsilon}^{e} \\
& \sigma_{e}=\sqrt{\left(E \bar{\varepsilon}^{e}\right)^{2}+3\left(\mu \kappa_{\varepsilon}^{e} r\right)^{2}}
\end{aligned}
$$

where, notably, the inclusion of pre-tension $\bar{\varepsilon}^{e}$ causes a difference from pure torsion in Liu and Soh (2016).

Thus, the total effective stress is

$$
\sigma_{e}^{t}=\sigma_{e}=\sqrt{\left.E \bar{\varepsilon}^{e}\right)^{2}+3\left(\mu \kappa_{\varepsilon}^{e} r\right)^{2}}
$$


The total effective strain is

$$
\varepsilon_{e}^{t, e p}=\left\{\left(\varepsilon_{e}^{e}+\varepsilon_{e}\right)^{2 \beta}+\left[l\left(\eta_{e}^{e}+\eta_{e}\right)\right]^{2 \beta}\right\}^{\frac{1}{2 \beta}}=\left\{\left[\bar{\varepsilon}+\frac{\left(\left|\kappa_{\varepsilon}^{e}\right|+\kappa_{\varepsilon}^{p}\right) r}{\sqrt{3}}\right]^{2 \beta}+\left[l\left(\kappa_{\eta}^{e}+\kappa_{\eta}^{p}\right)\right]^{2 \beta}\right\}^{\frac{1}{2 \beta}}
$$

where, the effect of pre-tension is represented by the appearance of $\bar{\varepsilon}$.

Based on Eqs. (3.12) and (3.15), the reference strain and stress are, respectively, expressed as

$$
\begin{aligned}
& \varepsilon_{0}^{t}=\left(\frac{\varepsilon_{e}^{t, e p}}{\varepsilon_{e}^{e}+\varepsilon_{e}}\right)^{\gamma_{\varepsilon}} \varepsilon_{Y}=\left\{1+\left[\frac{\sqrt{3} l\left(\kappa_{\eta}^{e}+\kappa_{\eta}^{p}\right)}{\sqrt{3} \bar{\varepsilon}+r\left(\left|\kappa_{\varepsilon}^{e}\right|+\kappa_{\varepsilon}^{p}\right)}\right]^{2 \beta}\right\}^{\frac{\gamma_{\varepsilon}}{2 \beta}} \varepsilon_{Y} \\
& \sigma_{0}^{t}=\left(\frac{\varepsilon_{e}^{t, e p}}{\varepsilon_{e}^{e}+\varepsilon_{e}}\right)^{\gamma_{\sigma}} \sigma_{Y}=\left\{1+\left[\frac{\sqrt{3} l\left(\kappa_{\eta}^{e}+\kappa_{\eta}^{p}\right)}{\sqrt{3} \bar{\varepsilon}+r\left(\left|\kappa_{\varepsilon}^{e}\right|+\kappa_{\varepsilon}^{p}\right)}\right]^{2 \beta}\right\}^{\frac{\gamma_{\sigma}}{2 \beta}} \sigma_{Y}
\end{aligned}
$$

where it should be kept in mind that the strain gradient is purely elastic through the present elasto-plastic decomposition and, therefore, at any moment $\kappa=\kappa_{\eta}^{e}+\kappa_{\eta}^{p}=\kappa_{\eta}^{e}$. Notably, the same assumption was made in Fleck et al. (2014).

The yield evolves when

$$
\sigma_{e}^{t}=\sigma^{t}
$$

where $\sigma^{t}$ can be given according to Eqs. (2.6) and (3.15)-(3.17).

Finally, the torque on the cross section of the wire can be expressed as

$$
T=2 \pi \mu \int_{0}^{a} \kappa_{\varepsilon}^{e} r^{3} d r
$$

\section{Results and discussions}

Figure 1 shows variation of normalized torque of wires with the surface strain. The dashed lines are resulted from the strain gradient elasto-plasticity (SGEP) in Liu and Soh (2016), whereas the red solid lines are obtained based on the simplified SGEP formulated in Section 2, where the elastic characteristic length scales are assumed to be zero and the plastic characteristic length scales are deemed the same. We can find that the simplified SGEP can provide almost identical results when compared with the version of SGEP by Liu and Soh (2016). This indicates that neglecting the of higher order stresses is proper at least under the present settings.

Figure 2 reveals strong dependence of the pre-tension wires on the wire radial size and the pre-tension strain $\bar{\varepsilon}$. The dot-dashed lines correspond to results without any pre-tension, i.e. $\bar{\varepsilon}=0$. For the wire of diameter $2 a=2 l$, let the pre-tension strain be $0 \%, 0.16 \%, 0.50 \%, 1.00 \%$ and $3.00 \%$, respectively, it is found that the yield strength decreases with the increasing pre-tension strain. For other sizes of wires, i.e. $2 a=4 l$ and $8 l$, the tendency is the same. On the other hand, for wires with equal pre-tension strain, the strong size effect can be observed. This is to say, the yield strength increases with the decreasing wire radius. For example, when $\bar{\varepsilon}=3.00 \%$, i.e. for the three dashed lines, the one corresponding to $2 a=2 l$ has the highest yield strength, whereas both to $2 a=4 l$ and $8 l$, are smaller. The influence of pre-tension is easy to explain based on Eqs. (3.15) and (3.16). The bigger $\bar{\varepsilon}$, the smaller $\varepsilon_{e}^{t, e p}$ will be. If the pre-tension deformation is purely elastic, after being transformed to torsion, the pure elastic deformation continues for a while before the yield initiation occurs, for example, when $\bar{\varepsilon}=0.07 \%$ and $2 a=2 l$. When $\bar{\varepsilon}=0.16 \%$ and $2 a=2 l$, there is some small plastic deformation during the tension stage. When torsion starts, the extra hardening effect of the strain gradient comes into play and, meanwhile, the pre-tension $\bar{\varepsilon}$ tends to weaken the gradient effect. The final yield strength depends on the 


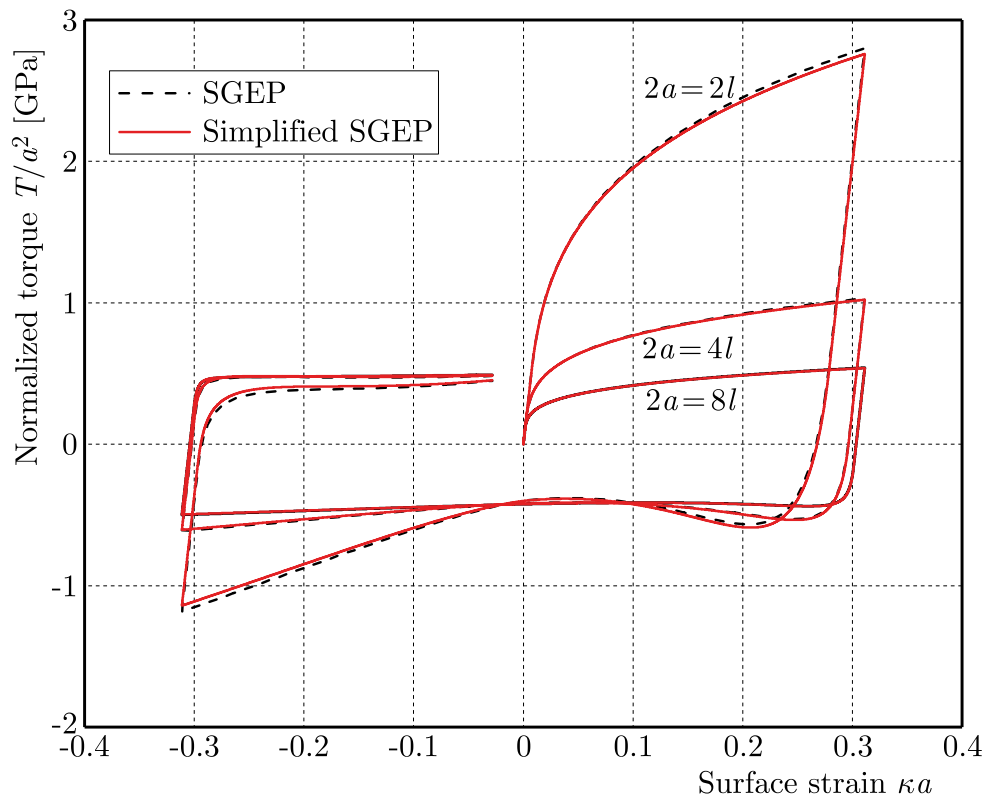

Fig. 1. Diagrams of the normalized torque $T / a^{3}$ versus the surface strain $\kappa a$ for torsions of polycrystalline $\mathrm{Cu}$ wires with diameter of $2 l, 4 l, 8 l$, respectively. Parameters are kept the same as those in Liu and Soh (2016) except that the elastic lengths are set to be zero, i.e., $\sigma_{Y}=100 \mathrm{MPa}$, $E=118 \mathrm{GPa}, L_{0}=L_{1}=L_{2}=0, l=l_{0}=l_{1}=l_{2}=2.83 \mu \mathrm{m}, \nu=0.5, \gamma_{\sigma}=2 \gamma_{\varepsilon}=2 \beta=2.0, N=0.22$. Note that in Liu and Soh (2016), $L_{0}=L_{1}=L_{2}=5 \cdot 10^{-2} \mu \mathrm{m}$

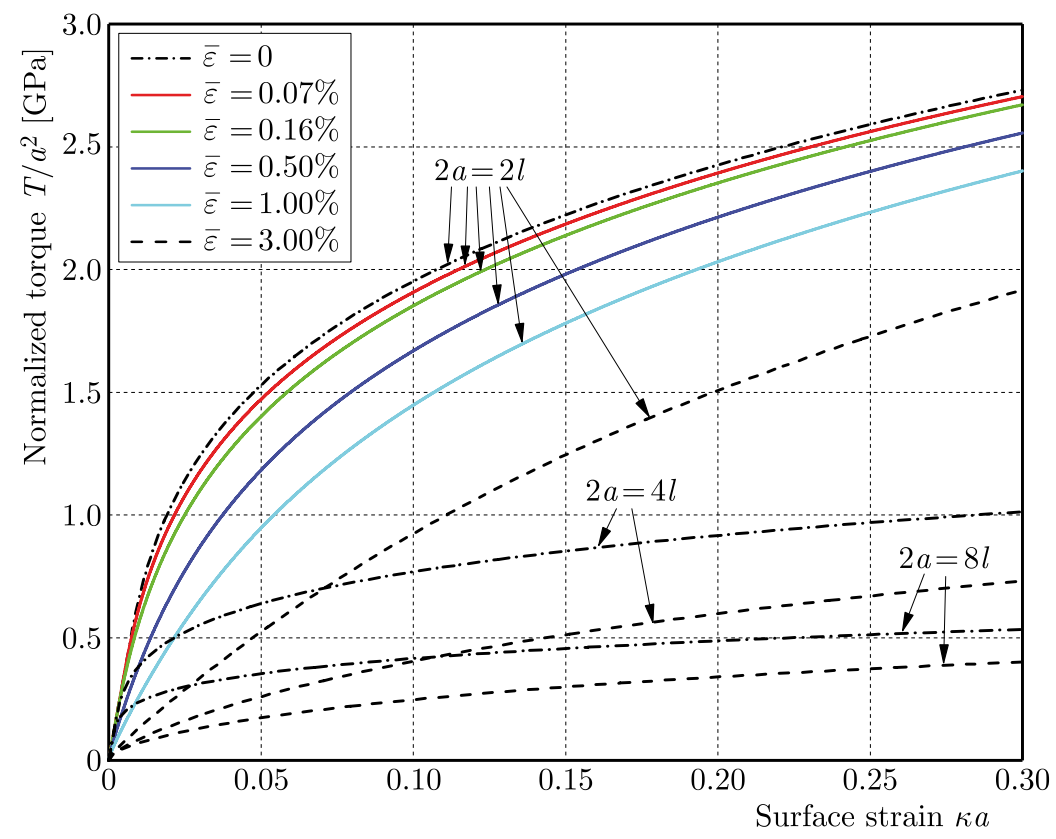

Fig. 2. Diagrams of the normalized torque versus the surface strain for wires of diameters $2 l, 4 l, 8 l$, respectively, and with different degrees of pre-tension, i.e. $\bar{\varepsilon}=0,0.07 \%, 0.16 \%, 0.50 \%, 1.00 \%$ and $3.00 \%$, respectively

competition between them. $\bar{\varepsilon}=0.16 \%$ is relatively small and, therefore, the extra hardening effect becomes dominant now. While for $\bar{\varepsilon}=0.5 \%$ and above, the influence of pre-tension becomes more important, and the yield evolution happens at the very beginning of torsion.

Another important notice from Fig. 2 is that dependence on the pre-tension exists in the size effect observed in pure torsion tests like in Fleck et al. (1994) and Dunstan et al. (2009). 
For example, the wire of size $2 a=2 l$ with $\bar{\varepsilon}=3.00 \%$ has a lower yield strength than that of size $2 a=4 l$ without any pre-tension, when the surface strain is smaller than 0.05 . It is also lower than that of $2 a=8 l$ without any pre-tension, when the surface strain is 0.025 and under. From this study, we conclude that the size effect related to the strain gradient is strongly influenced by the pre-tension that wires have experienced before torsion.

The competition between gradient-related extra hardening and pre-tension, which is aforementioned, can be shown more clearly in Fig. 3. During the torsion stage, at material points closer to the wire central axis, the extra hardening effect due to strain gradient is stronger, which decreases quickly with increasing $r$. On the other hand, for a fixed radial position $r$, the current yield strength $\sigma^{t}$ is strongly dependent on the pre-tension $\bar{\varepsilon}$. When the pre-tension is pretty large, for example, $\bar{\varepsilon}=3.00 \%, \sigma^{t}$ approximately shows a homogeneous distribution with respect to $r$. Additionally, the yield strength with $\bar{\varepsilon}=0$ is higher than that with $\bar{\varepsilon}=3.00 \%$ when $r \in(0,4.97) \mu \mathrm{m}$, and vice versa beyond this range. Similarly, the yield strength with $\bar{\varepsilon}=0.07 \%$ is higher than that with $\bar{\varepsilon}=3.00 \%$ when $r \in(0,2.57) \mu \mathrm{m}$, and vice versa beyond this range. This kind of phenomenon is actually the competition between the gradient extra hardening and the pre-tension strain hardening. From this viewpoint, again, we argue that the deformation history experienced by wires before torsion strongly influences the torsional yield evolution.

(a)

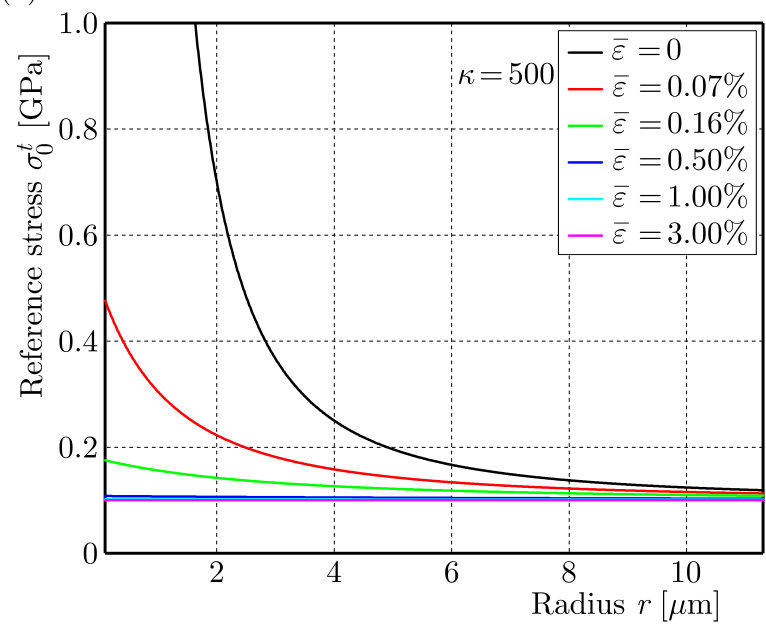

(b)

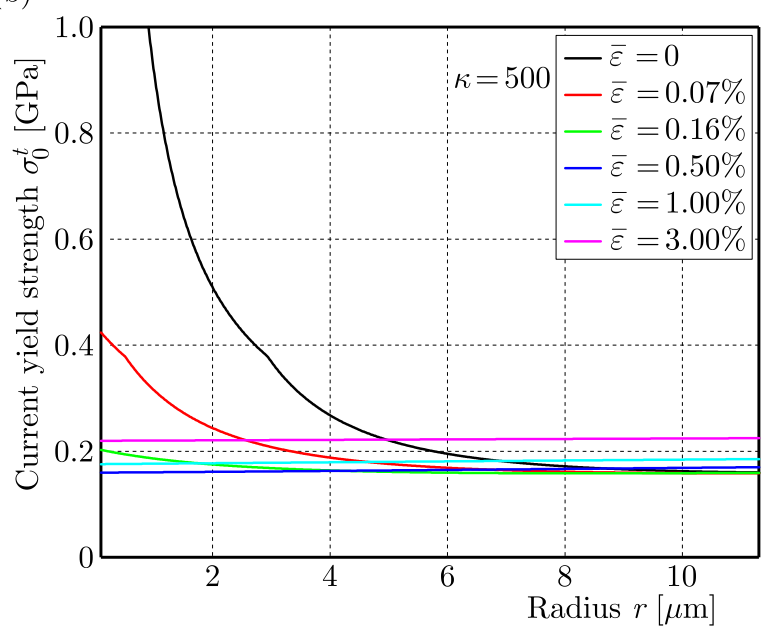

Fig. 3. Reference stress $\sigma_{0}^{t}$ (a) and current yield strength $\sigma^{t}$ (b) versus radius $r$ for torsion of wires with the pre-tension strain $0,0.07 \%, 0.16 \%, 0.50 \%, 1.00 \%$ and $3.00 \%$, respectively, when the twist increases monotonically to $\kappa=500$

The combination of the pre-tension strain hardening and the following gradient-related extra hardening is also analyzed at the position $r=a$, i.e. the wire surface, as shown in Fig. 4 . Since the tensile deformation is kept when torsion begins later on, for a nonzero pre-tension, the effective stress $\sigma_{e}^{t}$ already has a nonzero value which is inherited from the pre-tension. Furthermore, the initial value of $\sigma_{e}^{t}$ increases with the increasing pre-tension strain $\bar{\varepsilon}$. Of course, the curve without pretension, i.e. $\bar{\varepsilon}=0$, starts from zero initial effective stress. Once the torsion begins, however, the effective stress shows a profound inverse dependence on the pre-tension. For example, when the surface strain is 0.05 , the wire with a higher pre-tension strain has a lower effective stress. This can be explained as follows. After the torsion starts, the extra hardening due to strain gradient comes into play. According to Eq. (3.16), the current yield strength is related to the factor $\sqrt{3} l \kappa /(\sqrt{3} \bar{\varepsilon}+r \kappa)$, which decreases with the increasing $\bar{\varepsilon}$. In Fig. 4 , the wire diameter is on the same level of plastic characteristic length $l$, thus the gradient extra hardening is profound and even dominant in this case. As a result, the extra hardening takes over strain hardening and dominates the evolution of the current yield strength. 


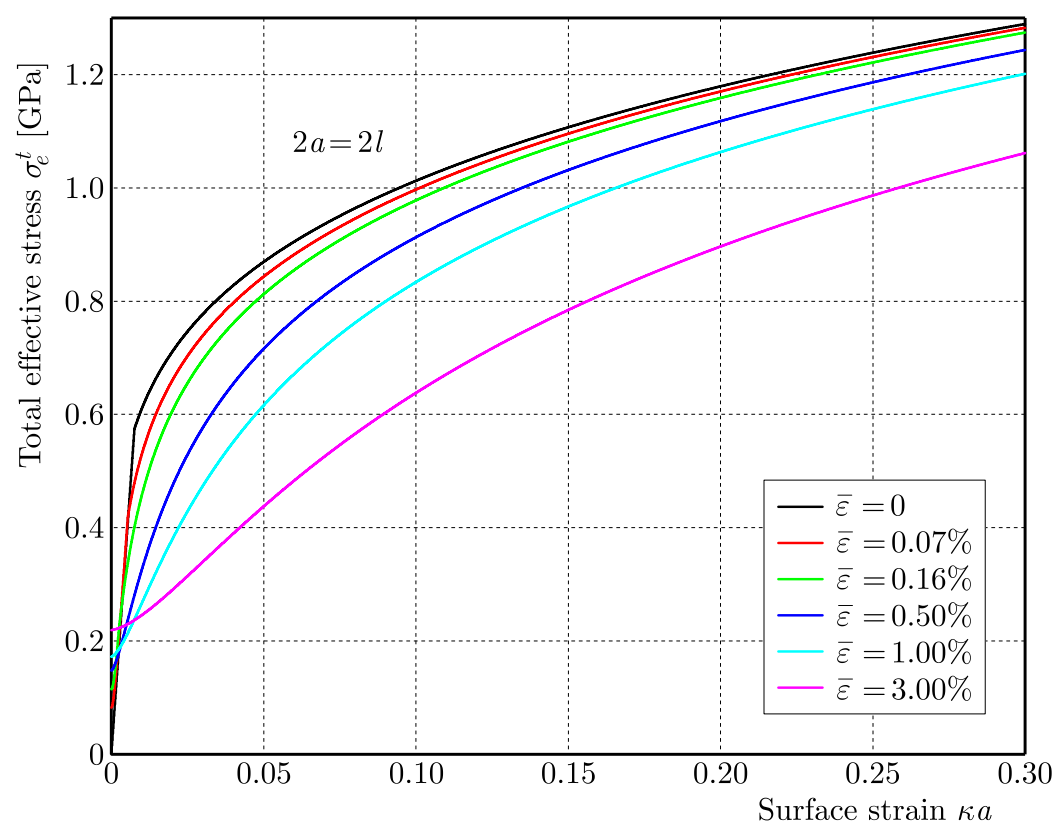

Fig. 4. The total effective stress $\left.\sigma_{e}^{t}\right|_{r=a}$, versus the surface strain in the wire of diameter $2 a=2 l$ and with the pre-tension strain $0,0.07 \%, 0.16 \%, 0.50 \%, 1.00 \%$ and $3.00 \%$, respectively
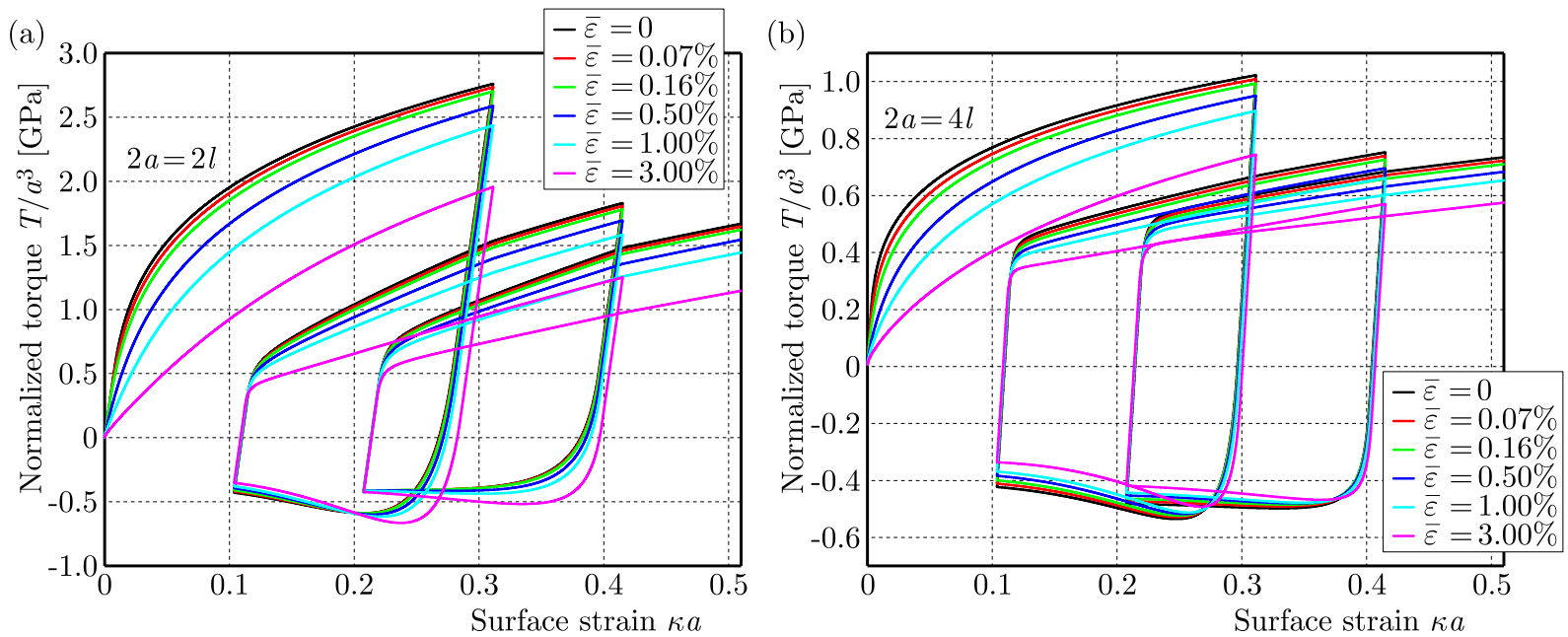

Fig. 5. Evolution of the normalized torque $T / a^{3}$ versus the surface strain under various pre-tension strains and wire diameters: (a) $2 a=2 l$, (b) $2 a=4 l$ and (c) $2 a=8 l$

The cyclic response is investigated in Fig. 5, where wires with different diameters and various pre-tension strains are simulated. As studied in Liu and Soh (2016), both the anomalous Bauschinger effect and plastic softening may occur, which can be predicted by the proposed strain gradient elasto-plasticity. Kiener et al. (2010) experimentally observed similar phenomena. From Fig. 5, the anomalous Bauschinger effect appears when the wire diameter is $2 a=2 l$, but disappears in thicker wires, showing an explicit size effect. The pre-tension tends to weaken the dependence of wire diameter or, in other words, the gradient-related extra hardening, which can be observed for variable wire diameter. The reason has been given before, namely due to the competition mechanism between gradient extra hardening and pre-tension strain hardening. Plastic softening, which corresponds to the shrinkage of the yield surface, are commonly observed for all wire sizes and pre-tension diameters. This is due to the argument that strain gradient is purely elastic under the present elasto-plastic decomposition. Meanwhile, it is influenced by the 
degree of extra hardening. For a larger wire diameter, the gradient extra hardening is weaker and, therefore, the plastic softening also weakens, but remains observable. On the other hand, both the anomalous Bauschinger effect and plastic softening quickly becomes weaker and weaker with increasing loading cycles. This is because in each cycle some plastic strain is produced and remains permanent, whereas no permanent strain gradient appears. As a result, cycle by cycle, the gradient-related extra hardening weakens continuously according to Eq. (3.16).

\section{Conclusions}

The tension-torsion of microscale copper wires is investigated based on the proposal of a simplified strain gradient elasto-plasticity. In the present theory, elastic lengths and, therefore, higher order stress are excluded, and no geometric decomposition of strain gradient has been introduced, leading to the nececeity of a single plastic characteristic length. The numerical results by this simplified model agree very well with those by the original theory in Liu and Soh (2016).

Torsional responses of wires with pre-tension show some important differences when compared with their counterparts under pure torsions. For a fixed-sized wire, with increasing pretension strain, the torsional yield strength decreases under monotonic torsion, and shows a weak anomalous Bauschinger effect and plastic softening under cyclic torsion. Particularly, for monotonic torsions, the yield strength of wires with a larger diameter and larger pre-tension strain can be significantly higher than that of wires with a smaller diameter and smaller pre-tension strain within a certain range of torsion surface strains. This indicates strong coupling between the size effect due to the gradient-related extra hardening and the effect of pre-tension strain hardening. The latter tends to weaken the former. This can be an informative hint to the controversial issues among experimental data in torsion tests by different research groups. The torsion yield strength strongly depends on the manufacturing history of wires.

Additionally, during the torsion stage of wires with pre-tension, there may or may not be an initial elastic stage. Furthermore, the elastic range also depends on the magnitude of pre-tension strain. For a wire with pretty larger pre-tension strains, e.g. $3.00 \%$, the wire may keep evolving plastically from the very beginning of torsion. This can be explained by considering the following two factors. One is that the pre-tension weakens the gradient-related hardening appearing in the torsion stage, and the other is that large enough pre-tension may already lead to considerable plastic deformations before torsion starts.

Last but not least, we have taken copper wires as an example in this study. Nevertheless, the present model is deemed quite general and also works for other metal materials. It is essentially material-dependent for the anomalous Bauschinger and plastic softening effect to appear or not, both of which may be captured by the proposed theory.

\section{Acknowledgements}

The work of X.B. Ma and J.X. Liu was supported by the National Science Foundation of China (Grant No. 11672119 and 11972174), Jiangsu Science Fund for Youth (BK20140520), the Jiangsu province and Jiangsu University Specially-Appointed Professor grants.

\section{References}

1. Bardella L., Panteghini A., 2015, Modelling the torsion of thin metal wires by distortion gradient plasticity, Journal of the Mechanics and Physics of Solids, 78, 467-492

2. Chakravarthy S.S., Curtin W.A., 2011, Stress-gradient plasticity, Proceedings of the National Academy of Sciences of the United States of America, 108, 38, 15716-15720 
3. Dunstan D.J., Ehrler B., Bossis R., Joly S., P'Ng K.M.Y., Bushby A.J., 2009, Elastic limit and strain hardening of thin wires in torsion, Physical Review Letters, 103, 15, 155501, DOI: 10.1103/PhysRevLett.103.155501

4. Eleftheriadis I., Neuhäuser H., Aifantis E., 2012, Torsional prestrain gradient and size dependence of initial yield for $<100>\mathrm{Cu}-\mathrm{Mn}$ single crystals in tension, Journal of the Mechanical Behavior of Materials, 21, 3-4, 95-99

5. Fertig R.S., Baker S.P., 2009, Simulation of dislocations and strength in thin films: a review, Progress in Materials Science, 54, 6, 874-908

6. Fleck N.A., Hutchinson J.W., 1997, Strain gradient plasticity, Advances in Applied Mechanics, 33, 295-361

7. Fleck N.A., Hutchinson J.W., 2001, A reformulation of strain gradient plasticity, Journal of the Mechanics and Physics of Solids, 49, 10, 2245-2271

8. Fleck N.A., Hutchinson J.W., Willis J.R., 2014, Strain gradient plasticity under non-proportional loading, Proceedings of the Royal Society, 470, 20140267

9. Fleck N.A., Muller G., Ashby M., Hutchinson J., 1994, Strain gradient plasticity: theory and experiment, Acta Metallurgica et Materialia, 42, 2, 475-487

10. FleCK N.A., Willis J.R., 2009, A mathematical basis for strain-gradient plasticity theory. Part II: Tensorial plastic multiplier, Journal of the Mechanics and Physics of Solids, 57, 7, 1045-1057

11. Forest S., Sievert R., 2003, Elastoviscoplastic constitutive frameworks for generalized continua, Acta Mechanica, 160, 1-2, 71-111

12. Gao H., Huang Y., 2001, Taylor-based nonlocal theory of plasticity, International Journal of Solids and Structures, 38, 15, 2615-2637

13. Gao H., HuAng Y., 2003, Geometrically necessary dislocation and size-dependent plasticity, Scripta Materialia, 48, 2, 113-118

14. Gudmundson P., 2004, A unified treatment of strain gradient plasticity, Journal of the Mechanics and Physics of Solids, , 52, 6, 1379-406

15. Idiart M.I., Fleck N.A., 2010, Size effects in the torsion of thin metal wires, Modelling and Simulation in Materials Science and Engineering, 18, 1, 015009

16. Janssens K.G.F., 2018, Proportionally and non-proportionally perturbed fatigue of stainless steel, International Journal of Fatigue, 110, 42-48

17. Kiener D., Motz C., Grosinger W., Weygand D., Pippan R., 2010, Cyclic response of copper single crystal micro-beams, Scripta Materialia, 63, 5, 500-503

18. Liu D., He Y., Dunstan D.J., Zhang B., Gan Z., Hu P., Ding H., 2013a, Anomalous plasticity in the cyclic torsion of micron scale metallic wires, Physical Review Letters, 110, 24, 244301, DOI: 10.1103/PhysRevLett.110.244301

19. Liu D., He Y., Dunstan D.J., Zhang B., Gan Z., Hu P., Ding H., 2013b, Toward a further understanding of size effects in the torsion of thin metal wires: an experimental and theoretical assessment, International Journal of Plasticity, 41, 1, 30-52, DOI: 10.1016/j.ijplas.2012.08.007

20. Liu D., He Y., Shen L., Lei J., Guo S., Peng K., 2015, Accounting for the recoverable plasticity and size effect in the cyclic torsion of thin metallic wires using strain gradient plasticity, Materials Science and Engineering: A, 647, 84-90, DOI: 10.1016/j.msea.2015.08.063

21. LiU J., SoH A. K., 2016, Strain gradient elasto-plasticity with a new Taylor-based yield function, Acta Mechanica, 227, 10, 3031-3048

22. Nicola L., Xiang Y., Vlassak J., der Giessen E.V., Needleman A., 2006, Plastic deformation of freestanding thin films: experiments and modeling, Journal of the Mechanics and Physics of Solids, 54, 10, 2089-2110 
23. Nix W.D., GaO H., 1998, Indentation size effects in crystalline materials: a law for strain gradient plasticity, Journal of the Mechanics and Physics of Solids, 46, 3, 411-425

24. Ozhoga-Maslovskaja O., Naumenko K., Altenbach H., Prygorniev O., 2015, Micromechanical simulation of grain boundary cavitation in copper considering non-proportional loading, Computational Materials Science, 96, 178-184

25. Pejkowski L., 2017, On the material's sensitivity to non-proportionality of fatigue loading, Archives of Civil and Mechanical Engineering, 17, 3, 711-727

26. Stölken J.S., Evans A.G., 1998, A microbend test method for measuring the plasticity length scale, Acta Materialia, 46, 14, 5109-5115

27. Xiang Y., Vlassak J., 2006, Bauschinger and size effects in thin-film plasticity, Acta Materialia, 54, 20, 5449-5460

28. Yang Y., Vormwald M., 2017, Fatigue crack growth simulation under cyclic non-proportional mixed mode loading, International Journal of Fatigue, 102, 37-47

Manuscript received January 14, 2019; accepted for print June 17, 2019 